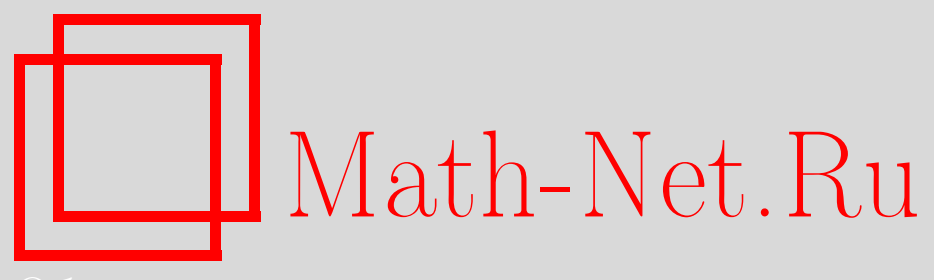

С. В. Шапошников, О внутренних оценках соболевских норм решений эллиптических уравнений, Матем. заметки, 2008, том 83, выпуск 2, 316-320

DOI: https://doi.org/10.4213/mzm4423

Использование Общероссийского математического портала Math-Net.Ru подразумевает, что вы прочитали и согласны с пользовательским соглашением http://www . mathnet.ru/rus/agreement

Параметры загрузки:

IP: 54.198 .55 .26

26 апреля 2023 г., 18:31:10

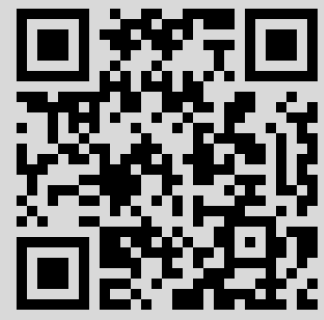




\section{О внутренних оценках соболевских норм решений эллиптических уравнений}

\section{С. В. Шапошников}

В этой работе изучаются решения $u \in W^{q, 1}(\Omega)$ эллиптического уравнения

$$
\partial_{x_{i}}\left(a^{i j} \partial_{x_{j}} u+b^{i} u\right)-c^{i} \partial_{x_{i}} u-d u=f-\partial_{x_{i}} e^{i}
$$

на ограниченной области $\Omega \subset \mathbb{R}^{n}$, причем $q \geqslant 2$. Уравнение (1) понимается в смысле интегрального тождества

$$
\int_{\Omega} \varphi_{x_{i}}\left(a^{i j} u_{x_{j}}+b^{i} u+e^{i}\right)+\varphi\left(c^{i} u_{x_{i}}+d u+f\right) d x=0
$$

для каждой функции $\varphi \in C_{0}^{1}(\Omega)$, где ведется суммирование по повторяющимся индексам. Для коэффициентов $a^{i j}, b, c$ и $d$ уравнения (1) выполняются следующие условия $\mathrm{H}(q)$ :

(a) $a^{i j} \in C^{0, \delta}(\Omega), a^{i j}=a^{j i}$ и существуют такие константы $m, M>0$, что

$$
m|y|^{2} \leqslant \sum_{i, j} a^{i j}(x) y_{i} y_{j} \leqslant M|y|^{2} \quad \text { для любых } \quad x \in \Omega, y \in \mathbb{R}^{n} ;
$$

(b) $b, c \in L^{n}(\Omega)$ и $d \in L^{n / 2}(\Omega)$ при $2 \leqslant q<n$;

(c) $b, c \in L^{s}(\Omega)$ и $d \in L^{s / 2}(\Omega)$ для некоторого $s>n$ при $2 \leqslant q=n$;

(d) $b, c \in L^{q}(\Omega), d \in L^{n q /(n+q)}(\Omega)$ при $q>n$.

Наша цель - получить оценку $W^{q, 1}$-нормы решения уравнения (1) на внутренней области $\Omega^{\prime} \subset \Omega$ через нормы функций $e, f$ и $L^{1}$-норму решения на большей области $\Omega$. Эта оценка является уточнением результата, который был сформулирован Морри в его книге [1; с. 156] не вполне корректным образом: с $\Omega^{\prime}=\Omega$, что неверно, например, для уравнения Лапласа на круге. Доказательство оценки Морри с исследованием зависимости константы от коэффициентов было дано в [2], где допущена такая же неточность, как и в книге Морри. Рассуждения из [2] будут верными для решений с нулевым граничным условием. Мы будем предполагать несколько более сильные условия на коэффициенты уравнения, чем в [1] и [2].

Через $W^{q, 1}(\Omega)$ обозначим соболевское пространство функций, которые принадлежат $L^{q}(\Omega)$ вместе с их обобщенными частными производными первого порядка. Это пространство наделяется стандартной нормой

$$
\|f\|_{W^{q, 1}(\Omega)}:=\|f\|_{q, 1}:=\|f\|_{L^{q}(\Omega)}+\|\nabla f\|_{L^{q}(\Omega)},
$$

где $\|\cdot\|_{L^{q}(\Omega)}=\|\cdot\|_{q}$ обозначает $L^{q}(\Omega)$-норму скалярных или векторных функций. Через $W^{q,-1}(\Omega)$ обозначим сопряженное пространство к $W^{q^{\prime}, 1}(\Omega)$, где $q^{\prime}=q(q-1)^{-1}$, а через $\|\cdot\|_{W^{q,-1}}:=\|\cdot\|_{q,-1}$ обозначим норму этого пространства. Пусть $C^{0, \delta}(\Omega)$ - пространство всех функций (возможно, векторных или матричных) на $\Omega$, являющихся гёльдеровыми порядка $\delta$. Для матричнозначного отображения $A$ на $\Omega$ положим

$$
\|A\|_{C^{0, \delta}}:=\sup _{x \in \Omega}\|A(x)\|+\sup _{x, y \in \Omega} \frac{\|A(x)-A(y)\|}{|x-y|^{\delta}} .
$$

Работа выполнена при поддержке Российского фонда фундаментальных исследований (грант № 07-01-00-536), ГФЕН (грант № 06-01-39-003), DFG 436 RUS 113/343/0(R) и SFB 701 при университете Билефельда.

(C) С. В. ШАПошников, 2008 
При $\gamma \geqslant 1, r>0$ и $g \in L^{\gamma}(\Omega)$, положим

$$
\Theta_{\gamma, g}(r)=\sup _{x \in \Omega}\left(\int_{B(x, r) \cap \Omega}|g(y)|^{\gamma} d y\right)^{1 / \gamma} .
$$

Через $B(x, r)$ обозначаем шар с центром в $x$ и радиусом $r$, а через $|B(x, r)|$ - его объем.

Напомним неравенство Юнга. Пусть $x>0, y>0, \varepsilon>0, \alpha>0, \beta>0, \alpha^{-1}+\beta^{-1}=1$, тогда

$$
x y \leqslant \varepsilon \frac{x^{\alpha}}{\alpha}+\varepsilon^{1-\beta} \frac{y^{\beta}}{\beta} .
$$

Теорема 1. Предположим, что $\Omega \subset \mathbb{R}^{n}$ - ограниченная область и для коэффичиентов $a^{i j}, b, c u d$ уравнения (1) выполняются условия $\mathrm{H}(q)$, причем $q \geqslant 2$. Пусть $e \in L^{q}(\Omega) u f \in L^{p}(\Omega)$, где $p=n q /(n+q)$ nри $q \neq n u p>n / 2$ при $q=n$. Тогда, если функиия $u \in W^{q, 1}(\Omega)$ является решением уравнения (1) на $\Omega$, то для всякой области $\Omega^{\prime}$ с замыканием в $\Omega$ выполняется оценка

$$
\|u\|_{W^{q, 1}\left(\Omega^{\prime}\right)} \leqslant C\left(\|u\|_{L^{1}(\Omega)}+\|e\|_{L^{q}(\Omega)}+\|f\|_{L^{p}(\Omega)}\right),
$$

где константа $C$ зависит от $\Omega, \Omega^{\prime}, n, m, M, q,\|A\|_{C^{0, \delta}}$ и от величин $\Theta_{n, b}, \Theta_{n, c}, \Theta_{n / 2, d}$ при $2 \leqslant q<n$, от числа s и норм $\|b\|_{L^{s}},\|c\|_{L^{s}},\|d\|_{L^{s / 2}}$ при $q=n$ и норм $\|b\|_{L^{q}},\|c\|_{L_{q}}$, $\|d\|_{L^{n q /(n+q)}}$ npu $q>n$.

ДоказатеЛьство. Пусть $B=B(a, r)$ - шар с замыканием в $\Omega$, причем $0<r<1$, и пусть функция $\zeta$ из $C_{0}^{\infty}(B)$ такова, что $0 \leqslant \zeta \leqslant 1, \zeta(x)>0$ внутри $B$ и $\zeta(x)=1$ на шаре $B(a, r / 2)$, причем

$$
J(s):=\sup _{x}|\nabla \zeta(x)| \zeta(x)^{-s}+\sup _{i, j, x}\left|\partial_{x_{i}} \partial_{x_{j}} \zeta(x)\right| \zeta(x)^{-s}<\infty, \quad 0<s<1 .
$$

Эти условия легко обеспечить, взяв $\zeta(x)=\psi(|x| / r), \psi \in C_{0}^{\infty}(\mathbb{R}), 0 \leqslant \psi \leqslant 1, \psi(y)=0$ при $|y| \geqslant 1, \psi(y)>0$ при $|y|<1, \psi(y)=1$ при $|y| \leqslant 1 / 2$ и $\psi(y)=\exp \left[\left(y^{2}-1\right)^{-1}\right]$ вблизи точек -1 и 1 . Всюду в теореме мы будем использовать в качестве срезающей функции только такую функцию $\zeta$.

1. Докажем теорему при $q=2$. Для этого оценим $\|u \zeta\|_{W^{2,1}(B)}$. Домножим уравнение (1) на функцию $u \zeta^{2}$ и проинтегрируем. Имеем

$$
\begin{aligned}
\int_{\Omega}\left[a^{i j} u_{x_{j}} u_{x_{i}} \zeta^{2} d x+2 a^{i j} u_{x_{j}} u \zeta \zeta_{x_{i}}+b^{i} u_{x_{i}} u \zeta^{2}+2 b^{i} u^{2} \zeta_{x_{i}} \zeta\right. \\
\left.\left.+e^{i} u_{x_{i}} \zeta^{2}+2 e^{i} u \zeta \zeta_{x_{i}}+c^{i} u_{x_{i}} u \zeta^{2}+d u^{2} \zeta^{2}+f u \zeta^{2}\right)\right] d x=0
\end{aligned}
$$

Используя неравенство (2) при $\alpha=\beta=2$ с достаточно малым $\varepsilon>0$ и условие (а) из $\mathrm{H}(q)$, имеем

$$
\|\zeta \nabla u\|_{L^{2}}^{2} \leqslant C_{1}\left(\|u \nabla \zeta\|_{L^{2}}^{2}+\|b u \zeta\|_{L^{2}}^{2}+\|c u \zeta\|_{L^{2}}^{2}+\|\sqrt{\mid \overline{|d|}} u \zeta\|_{L^{2}}^{2}+\|e \zeta\|_{L^{2}}^{2}+\left\|f u \zeta^{2}\right\|_{L^{1}}\right),
$$

где $C_{1}=C_{1}(n, m, M)$. Оценим слагаемые в правой части неравенства по отдельности. Положим $t=n$ при $n>2$ и $t=(s+2) / 2$ при $n=2$. Ясно, что $2<t<s$ при $n=2$. Для того, чтобы оценить $\|g u \zeta\|_{L^{2}}$, где $g$ равно $b, c$ или $\sqrt{|d|}$, применим неравенство Гёльдера с параметром $t / 2$ и теорему вложения Соболева $W^{2,1} \subset L^{2 t /(t-2)}$. Имеем

$$
\|g u \zeta\|_{2} \leqslant\|g\|_{t}\|u \zeta\|_{2 t /(t-2)} \leqslant C(n, t)\|g\|_{t}\|u \zeta\|_{2,1}
$$

причем $\|g\|_{t}=\Theta_{n, g}(r)$, если $t=n,\|g\|_{t} \leqslant|B(a, r)|^{1-t / s}\|g\|_{s}$, если $2<t<s$. Применяя неравенство Гёльдера с показателями $p, p^{\prime}=p(p-1)^{-1}$, где $p=2 n(n+2)^{-1}$ при $n>2$ и 
$p>1$ при $n=2$, неравенство (2) с $\alpha=\beta=2$ и теорему вложения Соболева $W^{2,1} \subset L^{p^{\prime}}$, получаем

$$
\left\|f u \zeta^{2}\right\|_{1} \leqslant\|f \zeta\|_{p}\|u \zeta\|_{p^{\prime}} \leqslant C(n, p)\|f \zeta\|_{p}\|u \zeta\|_{2,1} \leqslant \varepsilon^{2}\|u \zeta\|_{2,1}^{2}+\varepsilon^{-2} C(n, p)^{2}\|f \zeta\|_{p}^{2} .
$$

Оценим $\|u \nabla \zeta\|_{L^{2}}$. Пусть $l=4(n+2)^{-1}$ при $n>2$ и $0<l<1$ при $n=2$. Заметим, что ввиду теоремы вложения Соболева $W^{2,1} \subset L^{(2-l) /(1-l)}$. Применяя неравенство Гёльдера, имеем

$$
\begin{aligned}
\|u \nabla \zeta\|_{2} & =\left\|u \zeta^{(2-l) / 2} \zeta^{-(2-l) / 2} \nabla \zeta\right\|_{2} \leqslant J\left(1-\frac{l}{2}\right)\left(\int_{B}|u \zeta|^{2-l}|u|^{l} d x\right)^{1 / 2} \\
& \leqslant J\left(1-\frac{l}{2}\right)\|u\|_{1}^{l / 2}\|u \zeta\|_{(2-l) /(1-l)}^{(2-l) / 2} \leqslant J\left(1-\frac{l}{2}\right) C(n, l)\|u\|_{1}^{l / 2}\|u \zeta\|_{2,1}^{(2-l) / 2} .
\end{aligned}
$$

Применив неравенство Юнга (2) с $\alpha=2 / l$ и $\beta=2 /(2-l)$, получаем

$$
\|u \nabla \zeta\|_{2} \leqslant \varepsilon\|u \zeta\|_{2,1}+\varepsilon^{-l /(2-l)} C(n, l)\|u\|_{L^{1}} .
$$

Согласно оценке (4) с $g=1$, имеем $\|u \zeta\|_{L^{2}(B(a, r))} \leqslant C(n, t)|B(a, r)|^{1 / t}\|u \zeta\|_{2,1}$. Подставляя полученные оценки в (3) и учитывая, что согласно формуле Лейбница $\|u \zeta\|_{2,1} \leqslant\|u \nabla \zeta\|_{2}+$ $\|\zeta \nabla u\|_{2}+\|u \zeta\|_{2}$, находим

$$
\begin{gathered}
\|u \zeta\|_{W^{2,1}(B)} \leqslant C_{2}\left(\|b\|_{L^{t}(B)}+\|c\|_{L^{t}(B)}+\left(\|d\|_{L^{t / 2}(B)}\right)^{1 / 2}+|B(a, r)|^{1 / t}+\varepsilon\right)\|u \zeta\|_{W^{2,1}(B)} \\
+C_{2} \varepsilon^{-1}\left(\|u\|_{L^{1}(B)}+\|e\|_{L^{2}(B)}+\|f\|_{L^{p}(B)}\right)
\end{gathered}
$$

где $C_{2}=C_{2}(n, p, t, m, M)$. Выбирая $r>0$ и $\varepsilon>0$ так, что

$$
C_{2}\left(\|b\|_{L^{t}(B(a, r))}+\|c\|_{L^{t}(B(a, r))}+\left(\|d\|_{L^{t / 2}(B(a, r))}\right)^{1 / 2}+|B(a, r)|^{1 / t}+\varepsilon\right)<\frac{1}{2},
$$

приходим к оценке $\|u \zeta\|_{2,1} \leqslant 2 C_{2} \varepsilon^{-1}\left(\|u\|_{1}+\|e\|_{2}+\|f\|_{p}\right)$. Используя гладкое разбиение единицы, ассоциированное с конечным покрытием области $\Omega^{\prime}$ шарами радиуса $r$ с замыканием в $\Omega$, получаем требуемую оценку.

2. Рассмотрим случай $2<q<n$. Как и выше, начнем с оценки $\|u \zeta\|_{W^{q, 1}(B)}$. Можно считать, что $A$ продолжено на все $\mathbb{R}^{n}$ с сохранением всех условий. Известно (см., например, [3], [4] или [5]; в последних двух работах условие на $A$ заметно ослаблено), что при достаточно большом $\lambda=\lambda\left(n, q, m, M,\|A\|_{C^{0, \delta}}\right)>0$ найдется такое число $N=N\left(n, q, m, M,\|A\|_{C^{0, \delta}}\right)>1$, что для всякой функции $w \in W^{q, 1}\left(\mathbb{R}^{n}\right)$ с компактным носителем функция $\mathscr{A} w=\partial_{x_{i}}\left(a^{i j} \partial_{x_{j}} w\right)-\lambda w$ удовлетворяет неравенству

$$
\|w\|_{W^{q, 1}\left(\mathbb{R}^{n}\right)} \leqslant N\|\mathscr{A} w\|_{W^{q,-1}\left(\mathbb{R}^{n}\right)} .
$$

Функция $w=\zeta и$ удовлетворяет уравнению

$$
\mathscr{A} w=-\lambda \zeta u+a^{i j} \partial_{x_{i}} \zeta \partial_{x_{j}} u+\partial_{x_{i}}\left(a^{i j} u \partial_{x_{j}} \zeta\right)-\zeta \partial_{x_{i}}\left(b^{i} u\right)+c^{i} \partial_{x_{i}} u \zeta+d u \zeta-\zeta \partial_{x_{i}} e^{i}+f \zeta .
$$

Согласно (5) нам достаточно оценить норму правой части в $W^{q,-1}(B)$ через указанные в теореме параметры. Перепишем правую часть (6) в удобном для дальнейшего виде:

$$
\begin{aligned}
\mathscr{A} w= & \partial_{x_{i}}\left(a^{i j} u \partial_{x_{j}} \zeta-b^{i} u \zeta-e^{i} \zeta\right)-\lambda \zeta u+a^{i j} \partial_{x_{i}} \zeta \partial_{x_{j}} u \\
& +b^{i} u \partial_{x_{i}} \zeta+c^{i} \partial_{x_{i}}(u \zeta)-c^{i} u \partial_{x_{i}} \zeta+d u \zeta+e^{i} \partial_{x_{i}} \zeta+f \zeta .
\end{aligned}
$$


Напомним, что $\left\|\partial_{x_{i}} h^{i}\right\|_{q,-1} \leqslant\|h\|_{q}$ для всякой векторно-значной функции $h \in L^{q}$ и согласно теореме вложения $L^{p} \subset W^{q,-1}$ при $p=n q(n+q)^{-1}$. Следовательно, норма правой части оценивается через выражение

$$
\begin{array}{r}
\left\|a^{i j} u \partial_{x_{j}} \zeta\right\|_{q}+\left\|b^{i} u \zeta\right\|_{q}+\left\|e^{i} \zeta\right\|_{q}+C(n, p)\left[\lambda\|\zeta u\|_{p}+\left\|a^{i j} \partial_{x_{i}} \zeta \partial_{x_{j}} u\right\|_{p}+\left\|b^{i} u \partial_{x_{i}} \zeta\right\|_{p}\right. \\
\left.+\left\|c^{i} \partial_{x_{i}}(u \zeta)\right\|_{p}+\left\|c^{i} u \partial_{x_{i}} \zeta\right\|_{p}+\|d u \zeta\|_{p}+\left\|e^{i} \partial_{x_{i}} \zeta\right\|_{p}+\|f \zeta\|_{p}\right]
\end{array}
$$

где $C=C(n, p)$ - константа из теоремы вложения. Заметим, что $\left\|a^{i j} u \partial_{x_{j}} \zeta\right\|_{q} \leqslant M\|u \nabla \zeta\|_{q}$ и $\left\|e^{i} \partial_{x_{i}} \zeta\right\|_{p} \leqslant\|\nabla \zeta\|_{n}\|e\|_{q}$. Применяя неравенство Гёльдера с показателями $n / q, n /(n-q)$ и показателями $(n+q) / q,(n+q) / n$, получаем

$$
\begin{aligned}
& \left\|b^{i} u \zeta\right\|_{q}+C(n, p)\left[\left\|b^{i} u \partial_{x_{i}} \zeta\right\|_{p}+\left\|c^{i} \partial_{x_{i}}(u \zeta)\right\|_{p}+\left\|c^{i} u \partial_{x_{i}} \zeta\right\|_{p}\right] \\
& \leqslant C(n, q, p)\left[\left(\|b\|_{n}+\|c\|_{n}\right)\|u \zeta\|_{q, 1}+\left(\|b\|_{n}+\|c\|_{n}\right)\|u \nabla \zeta\|_{q}\right] .
\end{aligned}
$$

Неравенство Гёльдера с показателями $(n+q) / 2 q,(n+q) /(n-q)$ и теорема вложения Соболева дают

$$
\lambda\|\zeta u\|_{p}+\|d u \zeta\|_{p} \leqslant C(n, q)\left(\lambda|B(a, r)|^{2 q /(n+q)}+\|d\|_{n / 2}\right)\|u \zeta\|_{q, 1} .
$$

Остается оценить $\|u \nabla \zeta\|_{L^{q}}$. Пусть $l=q^{2} /(n q+q-n)$. Заметим, что $0<l<1$ и $(q-l) /(1-l)=n q /(n-q)$. Ввиду теоремы вложения Соболева и неравенства Гёльдера имеем

$$
\begin{aligned}
\|u \nabla \zeta\|_{q} & =\left\|\zeta^{1-l / q} u \zeta^{-1+l / q} \nabla \zeta\right\|_{q} \leqslant J\left(1-\frac{l}{q}\right)\left(\int_{B}|u \zeta|^{q-l}|u|^{l} d x\right)^{1 / q} \\
& \leqslant J\left(1-\frac{l}{q}\right)\|u\|_{1}^{l / q}\|u \zeta\|_{(q-l) /(1-l)}^{(q-l) / q} \leqslant J\left(1-\frac{l}{q}\right) C(n, l, q)\|u\|_{1}^{l / q}\|\zeta u\|_{q, 1}^{(q-l) / q} .
\end{aligned}
$$

Согласно неравенству Юнга (2) с $\alpha=q / l, \beta=q /(q-l)$ получаем

$$
\|u \nabla \zeta\|_{q} \leqslant \varepsilon\|u \zeta\|_{q, 1}+C(n, l, q, \varepsilon)\|u\|_{1} .
$$

Аналогично случаю $q=2$ выбираем $\varepsilon>0$ и $r>0$ достаточно малыми. Получаем

$$
\|u \zeta\|_{q, 1} \leqslant C_{3}\left(\|\nabla u \nabla \zeta\|_{p}+\|u\|_{1}+\|e\|_{q}+\|f\|_{p}\right) .
$$

Используя разбиение единицы, приходим к следующей оценке:

$$
\|u\|_{W^{q, 1}\left(\Omega^{\prime}\right)} \leqslant C_{4}\left(\|u\|_{W^{p, 1}\left(\Omega^{\prime \prime}\right)}+\|u\|_{L^{1}(\Omega)}+\|e\|_{L^{q}(\Omega)}+\|f\|_{L^{p}(\Omega)}\right),
$$

для всякой области $\Omega^{\prime} \subset \Omega^{\prime \prime} \subset \Omega$. Константа $C_{4}$ зависит от областей $\Omega^{\prime}, \Omega^{\prime \prime}, \Omega$ и указанных в условии теоремы величин. Положим $p_{k}=n p_{k-1}\left(n+p_{k-1}\right)$ и $p_{0}=q, p_{1}=p$. Так как $p_{k} / p_{k-1} \leqslant n /(n+1)$, найдется такое натуральное число $K$, что $p_{K} \leqslant 2$. Пусть $\left\{\Omega_{k}\right\}_{0 \leqslant k \leqslant K}$ - такое семейство областей, что $\Omega^{\prime} \subset \Omega_{k} \subset \Omega$ и $\Omega_{k} \subset \Omega_{k+1}$. Тогда по доказанному для каждого $0 \leqslant k \leqslant K$ выполняется оценка

$$
\|u\|_{W^{p_{k-1}, 1}\left(\Omega_{k-1}\right)} \leqslant C(k)\left(\|u\|_{W^{p_{k}, 1}\left(\Omega_{k}\right)}+\|u\|_{L^{1}(\Omega)}+\|e\|_{L^{q}(\Omega)}+\|f\|_{L^{p}(\Omega)}\right) .
$$

Так как $p_{k} \leqslant 2$ при $k=K$, оцениваем $\|u\|_{W^{p_{K}, 1}}$ через $\|u\|_{W^{2,1}}$ и используем уже доказанную оценку для случая $q=2$. Последовательно для каждого $k$ применяем неравенство (9) и получаем оценку $\|u\|_{W^{p_{0}, 1}\left(\Omega^{\prime}\right)}$. Это рассуждение завершает доказательство в случае $2<q<n$. Доказательство в случаях $q=n$ или $q>n$ проводится аналогично. Кроме того, доказательство при $q>n$ можно найти в [6].

Автор выражает глубокую благодарность профессору В. И. Богачеву за плодотворные обсуждения и замечания. 


\section{СПИСОК ЦИТИРОВАННОЙ ЛИТЕРАТУРЫ}

[1] C. B. Morrey, Multiple integrals in the calculus of variations, Grundlehren Math. Wiss., 130, Springer-Verlag, Berlin-Heidelberg-New York, 1966. [2] С. В. Шапошников, Матем. заметки, 79:3 (2006), 450-469. [3] A. Lunardi, V. Vespri, J. Differential Equations, 94:1 (1991), 1-40. [4] S.-S. Byun, Trans. Amer. Math. Soc., 357:3 (2005), 1025-1046. [5] N. V. Krylov, Comm. Partial Differential Equations, 32:1-3 (2007), 453-475. [6] В. И. Богачев, М. Рёкнер, С. В. Шапошников, Теория вероятн. и ее примен., 52:2 (2007), 240-270.

\section{С. В. Шапошников}

Поступило

Московский государственный

14.06.2007

университет им. М. В. Ломоносова

E-mail: shaposh.st@ru.net, starticle@mail.ru 